\title{
Testing Quantum Gravity via Cosmogenic Neutrino Oscillations
}

\author{
Joy Christiar* \\ Wolfson College, Oxford University, Oxford OX2 6UD, United Kingdom
}

(Dated: 20 September 2004)

\begin{abstract}
Implications of some proposed theories of quantum gravity for neutrino flavor oscillations are explored within the context of modified dispersion relations of special relativity. In particular, approximate expressions for Planck-scale-induced deviations from the standard oscillation length are obtained as functions of neutrino mass, energy, and propagation distance. Grounding on these expressions, it is pointed out that, in general, even those deviations that are suppressed by the second power of the Planck energy may be observable for ultra-high-energy neutrinos, provided they originate at cosmological distances. In fact, for neutrinos in the highest energy range of EeV to $\mathrm{ZeV}$, deviations that are suppressed by as much as the seventh power of the Planck energy may become observable. Accordingly, realistic possibilities of experimentally verifying these deviations by means of the next generation neutrino detectors - such as IceCube and ANITA - are investigated.
\end{abstract}

PACS numbers: 04.60.-m, 14.60.Pq, 95.85.Ry

\section{INTRODUCTION}

Despite many decades of intense efforts, the task of constructing a viable theory of quantum gravity remains largely a speculative enterprise. There is, of course, no shortage of approaches to quantum gravity, many with unprecedented mathematical sophistication and conceptual innovation, but more often than not they harbor mutually incompatible philosophies [1]. Worse still, the minuteness of the Planck length - or, equivalently, the enormity of the Planck energy - guarantees to frustrate any attempt to experimentally distinguish the better approaches from the worse. Amidst this dire state of affairs, in recent years a glimmer of hope has emerged, and blossomed into a sub-discipline of "the phenomenology of quantum gravity" 2]. This is based on the observation that, regardless of incompatibility in philosophies and diversity in theoretical details, several approaches to quantum gravity predict energy-momentum relations for elementary particles - in the semiclassical or effective theory limit - that differ from their special relativistic counterpart in somewhat similar manner $3,[4,5,6,6,8,6$ ]. In natural units, these modified dispersion relations can be expressed as:

$$
p^{2}+m^{2}=E^{2}\left[1-\sum_{n=1}^{\infty} \xi^{(n)} \frac{E^{n}}{m_{P}^{n}}\right],
$$

where $m_{P}$ is the Planck mass, and $\xi^{(n)}$ are dimensionless parameters, which do not necessarily vanish for all orders of suppression by the Planck energy, and depend in general on spin and helicity of the particles [10]. Clearly, away from the Planck regime (i.e., for $E \ll m_{P}$ ) these generalized relations effectively reproduce the familiar special relativistic dispersion relation: $E^{2}=p^{2}+m^{2}$.

*Electronic address: joy.christian@wolfson.oxford.ac.uk
Remarkably, despite the suppressions by the Planck energy, it was pointed out in Ref. 11] that delays in the times of arrival induced by these modified relations in photons originating at cosmological distances may be observable, at least up to the first-order of suppression. As anthologized in Ref. [2], since then much effort has been devoted to understanding the phenomenological implications of the relations such as (11). For example, based on observations of synchrotron radiation from the Crab nebula, together with a view that the modifications in (11) result from the existence of a preferred frame, strong bounds (of order $10^{-9}$ ) on the parameter $\xi^{(1)}$ have been obtained in Ref. 12] (see also [13, 14, 15, 16]). Thus, it appears that theories of quantum gravity that lead to a preferred frame, and as a result predict relations (1) with the parameter $\xi^{(1)}$ of order unity, have been ruled out by these observations. Moreover, it has been suggested in Ref. 17] that advance neutrino observatories such as ANTARES [18] may well provide enough sensitivity to put significant bounds even on the parameter $\xi^{(2)}$ in the relations (11), thereby constraining those theories of quantum gravity that predict only second and higher-order suppressions by the Planck energy (see also section 5.4 of Ref.[19]).

Suppose now that, instead of simply providing constraining bounds, eventually a genuine departure from the special relativistic dispersion relation is actually detected. Of course, that would be a tremendous boost for some of the approaches to quantum gravity, not to mention the much anticipated revolution it would finally bring about in physics. Inevitably, however, such a detection would also leave a great deal of ambiguity as to which of the proposed approaches to quantum gravity is truly compatible with the observed departure. For, as mentioned above, several approaches lead to essentially the same quantitative predictions for a departure from the special relativistic dispersion relation. It is therefore necessary to search for options other than those existing in the literature, particularly those that are more quantum mechanical in character. It is with this in mind 
that we explore here the implications of the relations (11) for neutrino flavor oscillations. To be sure, such a study has been initiated before within specific models 6. 20, 21, 22, 23, 24, 25, 26, 27]. Our aim here, however, is to investigate the generic relations (10), independently of any specific model. Moreover, since the linear suppression by the Planck energy seems to have been almost certainly ruled out [12], our concern here would be to point out that, in general, even those modified features of the standard theory of neutrino oscillations that are quadratically suppressed by the Planck energy may be observable for ultra-high-energy neutrinos, provided they have originated at cosmological distances. In fact, we shall show that, for such "cosmogenic" neutrinos in the highest energy range of $10^{18}$ to $10^{21} \mathrm{eV}$, modifications that are as minute as cubically, quartically, quintically, sextically, or even septically suppressed by the Planck energy may also become observable. Accordingly, we shall discuss realistic possibilities of verifying these modifications by means of the next generation neutrino detectors, such as IceCube 28] and ANITA 29].

To this end, in Section II we begin by briefly reviewing the standard theory of neutrino flavor oscillations, thereby stressing our preferred prescription (as opposed to the one widespread in textbooks) for arriving at the standard oscillatory phase factor. Then, in Section III, we evaluate the Planck scale modifications of the standard theory, induced by the generalized relations (1), and by a related relation proposed in Ref.[30]. Finally, before concluding in Section VI, we take up the issue of observability of these modifications in Sections IV and V.

\section{THE STANDARD THEORY OF NEUTRINO FLAVOR OSCILLATIONS}

The remarkable and quintessentially quantum phenomena of neutrino flavor oscillations are the result of the fact that neutrinos of definite flavor states $\left|\nu_{\alpha}\right\rangle, \alpha=e, \mu$, or $\tau$, are not particles of definite mass states $\left|\nu_{j}\right\rangle, j=1,2$, or 3. Rather, they are coherent superpositions of the definite mass states:

$$
\left|\nu_{\alpha}\right\rangle=\sum_{j} U_{\alpha j}^{*}\left|\nu_{j}\right\rangle
$$

with $U$ being the (time-independent) leptonic mixing matrix. By the same token, neutrinos of definite mass states are coherent superpositions of the definite flavor states:

$$
\left|\nu_{j}\right\rangle=\sum_{\beta} U_{\beta j}\left|\nu_{\beta}\right\rangle
$$

with the mixing matrix being subject to the unitarity constraint

$$
\sum_{j} U_{\alpha j}^{*} U_{\beta j}=\delta_{\alpha \beta}
$$

(The reference we shall largely follow here is Ref. 31], but see also Ref. 32] for a comprehensive review of the standard theory.) As a neutrino of definite flavor state $\left|\nu_{\alpha}\right\rangle$ originating, say, from a distant cosmological sourcepropagates through vacuum for a sufficiently long laboratory time, the heavier mass-eigenstates in the superposition, such as (2), lag behind the lighter ones, and, as a result, the neutrino ends up arriving at a terrestrial detector in an altogether different flavor state, say $\left|\nu_{\beta}\right\rangle$. The probability for this transition from one flavor state to another can be easily obtained as follows. In the rest frame of each $\left|\nu_{j}\right\rangle$, where the proper time is $\tau_{j}$, plane wave analysis leads to the Schrödinger equation

$$
i \frac{\partial}{\partial \tau_{j}}\left|\nu_{j}\left(\tau_{j}\right)\right\rangle=m_{j}\left|\nu_{j}\left(\tau_{j}\right)\right\rangle
$$

with a solution

$$
\left|\nu_{j}\left(\tau_{j}\right)\right\rangle=e^{-i m_{j} \tau_{j}}\left|\nu_{j}(0)\right\rangle,
$$

where $m_{j}$ is the eigenvalue of the mass-eigenstate $\left|\nu_{j}(0)\right\rangle$. For our purposes it is important to note that the phase factor $e^{-i m_{j} \tau_{j}}$ in the last equation is manifestly Lorentz invariant. In terms of the coordinate time $t$ and position $\vec{x}$ in the laboratory frame, this phase factor takes the familiar form

$$
e^{-i\left(E_{j} t-\vec{p}_{j} \cdot \vec{x}\right)},
$$

where $E_{j}$ and $\vec{p}_{j}$ are, respectively, the energy and momentum associated with the definite mass state $\left|\nu_{j}(0)\right\rangle$.

Now, neutrinos are highly relativistic particles-i.e., they propagate with speeds extremely close to the speed of light, which permits the convenient assumption $t \approx x$ $=L$, where $L$ is the distance traversed by neutrinos between production and detection. Moreover, assuming that the neutrinos are produced with the same energy $E$ regardless of which state $\left|\nu_{j}(0)\right\rangle$ they are in (and that $m_{j} \ll E$ ), up to the second order in $m_{j}$ the special relativistic dispersion relation gives the following expression for their momenta,

$$
p_{j}=\sqrt{E^{2}-m_{j}^{2}} \approx E-\frac{m_{j}^{2}}{2 E},
$$

which, along with the assumption $t \approx L$, reduces the phase factor in (7) to

$$
e^{-i \frac{m_{j}^{2}}{2 E} L}
$$

Here the assumed equality of energy for all $\left|\nu_{j}(0)\right\rangle$ may seem to go against Lorentz invariance, but it can be justified rigorously by noting that the quantum coherence we seek would be maintained only between wavepacket components associated with the same energy [33]. Consequently, in the laboratory frame, and up to the second order in $m_{j}$, the time evolution of the neutrino flavor 
state (2) - duly respecting the Lorentz invariance [34] is given by

$$
\begin{aligned}
\left|\nu_{\alpha}(t)\right\rangle & =\sum_{j} U_{\alpha j}^{*} e^{-i \frac{m_{j}^{2}}{2 E} L}\left|\nu_{j}(0)\right\rangle \\
& =\sum_{\beta} \sum_{j} U_{\alpha j}^{*} e^{-i \frac{m_{j}^{2}}{2 E} L} U_{\beta j}\left|\nu_{\beta}(0)\right\rangle .
\end{aligned}
$$

If we now restrict to the typical scenario of just two neutrino states of definite masses, $\left|\nu_{1}\right\rangle$ and $\left|\nu_{2}\right\rangle$, then the relevant unitary mixing matrix is simply a $2 \times 2$ submatrix of the general mixing matrix $U$ 31]. As a result, the transition probability for the neutrinos to "oscillate" from a given flavor state, say $\left|\nu_{\mu}(0)\right\rangle$, to another flavor state, say $\left|\nu_{e}(t)\right\rangle$, is given by

$$
\begin{aligned}
P_{\nu_{\mu} \rightarrow \nu_{e}}(E, L) & =\left|\left\langle\nu_{e}(0) \mid \nu_{\mu}(t)\right\rangle\right|^{2} \\
& =4\left|U_{\mu 2}\right|^{2}\left|U_{e 2}\right|^{2} \sin ^{2}\left(\frac{\Delta m^{2}}{4 E} L\right),
\end{aligned}
$$

where $\Delta m^{2} \equiv m_{2}^{2}-m_{1}^{2}>0$ is the difference in the squares of the two masses. Now, in terms of the mixing angle $\theta$, the quantity $4\left|U_{\mu 2}\right|^{2}\left|U_{e 2}\right|^{2}$ simply turns out to be equal to $\sin ^{2} 2 \theta$ (cf. 31] ). Using this, and a trigonometric identity, the transition probability can finally be recast in the following perspicuous form:

$$
P_{\nu_{\mu} \rightarrow \nu_{e}}(E, L)=\frac{1}{2} \sin ^{2} 2 \theta\left[1-\cos \left(\frac{\Delta m^{2}}{2 E} L\right)\right],
$$

where, incidentally (due to (8)),

$$
\frac{\Delta m^{2}}{2 E} \equiv \Delta p \equiv p_{1}-p_{2} \text {. }
$$

From this transition probability it is clear that the experimental observability of neutrino flavor oscillations is essentially determined by the quantum phase

$$
\Phi:=2 \pi \frac{L}{L_{O}},
$$

where

$$
L_{O}(E, m):=\frac{2 \pi}{\Delta p}=\frac{4 \pi E}{\Delta m^{2}}
$$

is the energy-dependent oscillation length. In particular, flavor changes would be observable whenever the propagation distance of the neutrinos, $L$, is of the order of the oscillation length, $L_{O}$. Therefore, in what follows, it would suffice to concentrate on these two variables.

\section{PLANCK SCALE CORRECTIONS TO THE OSCILLATION LENGTH}

Now, let us assume that the standard theory reviewed above remains essentially valid for ultra-high-energy neutrinos. It is then natural to wonder how the theory is generalized by approaches to quantum gravity that give rise to modifications of the form (1) of the standard dispersion relation $E^{2}=p^{2}+m^{2}$. The question can be easily answered by replacing the approximation (8) by

$$
p_{j} \approx E-\frac{m_{j}^{2}}{2 E}-\frac{\xi_{j}^{(1)}}{2} \frac{E^{2}}{m_{P}}-\left(\frac{\left(\xi_{j}^{(1)}\right)^{2}}{8}+\frac{\xi_{j}^{(2)}}{2}\right) \frac{E^{3}}{m_{P}^{2}},
$$

which follows from the modified dispersion relations (1) after the terms higher than second-order in $m_{j}$ and $m_{P}^{-1}$, as well as the terms involving $m_{j}^{2} / m_{P}$, are ignored. Here, following Refs. 6, 7, 20], we have assumed that the coefficients $\xi^{(1)}$ and $\xi^{(2)}$ in the expansion (11) may in general depend on the neutrino flavor (and hence on flavor-mixing, in accordance with Eq.(3)). The corresponding modified oscillation length, analogous to (15), is then given by

$$
\widetilde{L}_{O}\left(E, m, \xi^{(1)}, \xi^{(2)}\right):=\frac{2 \pi}{\Delta p}=\frac{2 \pi}{\frac{\Delta m^{2}}{2 E}+\Delta q},
$$

where we have set

$$
\Delta q \equiv \frac{\Delta \xi^{(1)}}{2} \frac{E^{2}}{m_{P}}+\left(\frac{\Delta\left(\xi^{(1)}\right)^{2}}{8}+\frac{\Delta \xi^{(2)}}{2}\right) \frac{E^{3}}{m_{P}^{2}},
$$

along with

$$
\begin{aligned}
& \Delta \xi^{(1)} \equiv \xi_{2}^{(1)}-\xi_{1}^{(1)} \\
& \Delta\left(\xi^{(1)}\right)^{2} \equiv\left(\xi_{2}^{(1)}\right)^{2}-\left(\xi_{1}^{(1)}\right)^{2}, \text { and } \\
& \Delta \xi^{(2)} \equiv \xi_{2}^{(2)}-\xi_{1}^{(2)}
\end{aligned}
$$

This is clearly an odd result. It implies that, according to various approaches to quantum gravity that lead to the modifications such as (1), flavor oscillations can occur even for neutrinos with negligible masses, or for massive neutrinos with degenerate mass-eigenstates [20]. The corresponding purely quantum gravity induced oscillation length - in either of the two cases - would be

$$
L_{Q G}\left(E, \xi^{(1)}, \xi^{(2)}\right)=\frac{2 \pi}{\Delta q}
$$

where $\Delta q$ is again given by (18). Of course, this curious conclusion depends on the above assumption that the coefficients $\xi^{(n)}$ may in general be flavor-dependent.

It is possible to avoid this conclusion altogether, however, within a different approach. Note that the modification (17) of the oscillation length arises from the replacement of the Lorentz-invariant quantum phase factor (7) by a generalized factor with $p_{j}$ given by (16). There are at least two possible interpretations of this generalized factor, depending on how the Lorentz invariance is treated in a given approach to quantum gravity: Either the relativity of inertial frames is taken as effectively broken in a theory, allowing the existence of a privileged frame, or it is taken as preserved, but Lorentz transformations are made to act non-linearly on the energy 
and momentum eigenstates of the theory. In the former case the conservations of energy and momentum are assumed to remain linear, whereas in the latter case they are deemed to be non-linear, affecting any experimental analysis 10]. There is, however, a third possibility in which the relativity of inertial frames is indeed generalized at the fundamental level, but without permitting a privileged reference frame, and without compromising the linearity of the conservations of energy and momentum. A theory incorporating this largely unappreciated possibility has been proposed in Ref. [30], which gives rise to the following generalization of the special relativistic dispersion relation:

$$
p^{2}+m^{2}=E^{2}\left[1-\frac{(E-m)^{2}}{m_{P}^{2}}\right] .
$$

There are several features of this generalization that are worth bringing out. To begin with, unlike the approximate nature of (11), the above expression is exact, with no other but a quadratic suppression by the Planck energy. Moreover, as it is supposed to be a part of truly fundamental theory replacing special relativity at the Planck scale, there are no parameters in the expression to be adjusted. And yet, independently of the Planck scale, in the rest frame of the particle it duly reproduces $E=m c^{2}$. What is more, the expression leads to a Planck scale induced modification of the neutrino flavor oscillations that turns out to be quite interesting in its own right. To appreciate this, note that according to (21), for $m \ll E$, and up to only the quadratic suppression by the Planck energy, the modified momentum of a neutrino of mass $m_{j}$ can be approximated as

$$
p_{j} \approx E-\frac{m_{j}^{2}}{2 E}+\frac{E^{2}}{m_{P}^{2}} m_{j}
$$

provided the terms involving the ratio $m_{j}^{2} / m_{P}$ are also deemed negligible. The corresponding modified oscillation length, analogous to (17), is then given by

$$
L_{O}^{\prime}(E, m):=\frac{2 \pi}{\Delta p}=\frac{2 \pi}{\frac{1}{2 E} \Delta m^{2}-\frac{E^{2}}{m_{P}^{2}} \Delta m},
$$

where $\Delta m^{2} \equiv m_{2}^{2}-m_{1}^{2}$ as before, and $\Delta m \equiv m_{2}-m_{1}$. For neutrinos with energy much smaller than the Planck energy this generalized oscillation length clearly reduces to the standard expression (15), whereas for neutrinos with energy approaching the Planck energy the second term in the denominator of (23) dominates, yielding

$$
L_{O}^{\prime}(E, m) \rightarrow L_{R R}(E, m):=\frac{2 \pi}{\Delta r}:=\frac{-2 \pi}{\Delta m} \frac{m_{P}^{2}}{E^{2}} .
$$

More significantly, and in sharp contrast with the peculiar implications of (17) discussed above, the generalized length (23) implies (quite sensibly) that there would be no flavor oscillations for massless particles, or for massive neutrinos with degenerate mass-eigenstates.

\section{OBSERVABILITY OF THE PLANCK SCALE INDUCED OSCILLATIONS}

Let us now address the question of experimental distinguishability of the two modified oscillation lengths above, (23) and (17), from each other, and from their special relativistic counterpart, (15). Beginning with the length (23), it is clear that the Planck scale induced modification would become significant in this case when

$$
\frac{\Delta m^{2}}{2 E} \sim \frac{E^{2}}{m_{P}^{2}} \Delta m
$$

or, equivalently, when

$$
L_{O} \equiv \frac{4 \pi E}{\Delta m^{2}} \sim L_{R R} \equiv\left|\frac{2 \pi}{\Delta r}\right|=\frac{2 \pi}{\Delta m} \frac{m_{P}^{2}}{E^{2}} .
$$

Of course, for those neutrinos of energy such that (25) is an exact equality, flavor oscillations would be washed out, providing a distinctive signature for the modified relation (21). More generally, the energy necessary to reveal deviations from the standard flavor oscillations would depend on the sensitivity with which the value of the mass splittings can be inferred in a given experiment. In experiments performed to date, this sensitivity ranges from $\Delta m^{2} \sim 1(\mathrm{eV})^{2}$, for short-baseline accelerator neutrinos, to $\Delta m^{2} \sim 10^{-11}(\mathrm{eV})^{2}$, for solar neutrinos [31]. These values can be easily calculated by noting from (12) that neutrino flavors oscillate as a function of $L / E$, and that $\Delta m^{2}$ reach for such oscillations is inversely proportional to this ratio:

$$
\Delta m^{2} \sim \frac{4 \pi E}{L}
$$

Substituting this reach into the condition (25) (along with the assumption $m_{2} \gg m_{1}$ ) yields

$$
L \sim \frac{\pi m_{P}^{4}}{E^{5}}
$$

This, then, is the necessary constraint between the neutrino energy $E$ and its propagation distance $L$, for detecting significant deviations from the standard flavor oscillations. For example, it can be easily calculated from this condition that the Planck scale deviations in the oscillation length, induced by the generalization (21), would be either observable, or can be ruled out, for neutrinos of energy $E \sim 10^{17} \mathrm{eV}$, provided that they have originated from sources located at some $10^{5}$ light-years away from the detector. According to (27), the corresponding confidence in the mass splittings $\Delta m^{2}$ would then be of the order of $10^{-10}(\mathrm{eV})^{2}$, which is comparable to that achieved for the solar neutrinos 31].

Turning now to the modified oscillation length (17), it is clear, once again, that the Planck scale induced modification would become significant in this case when

$$
\frac{\Delta m^{2}}{2 E} \sim \Delta q
$$


with $\Delta q$ given by (18), or, equivalently, when

$$
L_{O} \equiv \frac{4 \pi E}{\Delta m^{2}} \sim L_{Q G} \equiv \frac{2 \pi}{\Delta q} .
$$

Now, as we noted after the definition (15), for flavor changes to be observable at all, the propagation distance $L$ of the neutrinos must be about the same size as their oscillation length $L_{O}$. Moreover, it is easy to infer from (16) and (18) that $\Delta q$ is an expansion of the form

$$
\Delta q \approx \sum_{n=1} f_{n} \frac{E^{n+1}}{m_{P}^{n}}
$$

with $f_{n}$ being the functions of various splittings such as $\Delta \xi^{(n)}$, even when the order of suppression is kept arbitrary. Consequently, putting all of the above observations together, it is easy to see that the Planck scale deviations from the standard oscillation length would become significant in this case, for each order of suppression by the Planck energy, when

$$
L \sim \frac{2 \pi}{f_{n}} \frac{m_{P}^{n}}{E^{n+1}} .
$$

Now, up to linear suppression by the Planck energy, $f_{1} \equiv \Delta \xi^{(1)} / 2$, and the existing data from long-baseline accelerator neutrinos already imply that the splittings $\Delta \xi^{(1)}$ cannot be greater than $10^{-4}$ (using values from Ref. 31], for such terrestrial neutrinos $E \sim 10 \mathrm{GeV}$ and $L \sim 10^{3} \mathrm{~km}$, which, upon substitutions in (32), gives $\left.\Delta \xi^{(1)} \lesssim 10^{-4}\right)$. Unfortunately, since quantum interferences can only reveal relative phases of the interfering amplitudes, flavor oscillations can only be sensitive to the splittings such as $\Delta \xi^{(1)}$, and not to the underlying individual coefficients such as $\xi_{1}^{(1)}$ or $\xi_{2}^{(1)}$. On the other hand, provided cosmogenic neutrinos are at our disposal, remarkably strong bounds on the splittings $\Delta \xi^{(n)}$ - for at least up to the seventh order of suppression - can be obtained with reasonable neutrino energies.

To appreciate this, suppose we demand that the splittings $\Delta \xi^{(n)}$ be probed $\sigma$ times as deeply as the mass splittings $\sqrt{\Delta m^{2}}$; that is, suppose that

$$
\Delta \xi^{(n)} \sim \sigma \sqrt{\frac{\Delta m^{2}}{m_{e}^{2}}} \sim \sigma \sqrt{\frac{4 \pi E}{m_{e}^{2} L}},
$$

where $m_{e}$ is the electron mass (as standardly used in the neutrino experiments), and the last relation follows from (27). Then, assuming that all but the $n^{\text {th }}$ order coefficient $\xi_{j}^{(n)}$ in the expansion (11) are negligible (i.e., reducing $f_{n}$ to $\Delta \xi^{(n)} / 2$ ), the condition (32) becomes

$$
L \sim \frac{4 \pi m_{e}^{2}}{\sigma^{2}} \frac{m_{P}^{2 n}}{E^{2 n+3}},
$$

which specifies the constraint between the energy $E$ and propagation distance $L$ of neutrinos for probing the $n^{t h}$ order correction due to the modified dispersion relations
(11). For example, for the case of quadratic suppression by the Planck energy $(n=2)$, with $\sigma$ as stringent as $10^{-10}$, condition (34) dictates the values $E \sim 10^{16}$ $\mathrm{eV}$ and $L \sim 10^{10}$ light-years, giving $\Delta m^{2}$ reach $\sim 10^{-16}$ $(\mathrm{eV})^{2}$ and $\Delta \xi^{(2)}$ reach $\sim 10^{-24}$. These remarkable values may seem incredible, but it can be easily checked that the higher-order terms neglected in the expansion (16) (with $n \equiv 2$ ) continue to remain negligible even with these minute values for $\Delta m^{2}$ and $\Delta \xi^{(2)}$. On the other hand, neither condition (34) nor condition (28) necessitates $\Delta m^{2}$ to be as small as $10^{-16}(\mathrm{eV})^{2}$ for the quantum gravity effects to be observable. In fact, it can be as large as $10^{-4}(\mathrm{eV})^{2}$, or larger, for reasonable values of $E$ and $L$. For instance, for $E \sim 10^{19} \mathrm{eV}$ and $L \sim 10$ light-years, yielding $\Delta m^{2} \sim 10^{-4}(\mathrm{eV})^{2}$, the condition (34) (or (32)), with $n=2$, leads to $\Delta \xi^{(2)}$ reach as deep as $\sim 10^{-24}$.

Alternatively, one may wish to relinquish this high sensitivity of $\Delta \xi^{(n)}$ for the sake of probing deviations from $E^{2}=p^{2}+m^{2}$ that are suppressed by $m_{P}$ at a much higher order. As an extreme example, one can imagine a model in which quantum gravity induced deviations become significant only at the seventh order of suppression by the Planck energy. Then, with $\sigma$-setting as high as $10^{+8}$, condition (34), with $n=7$, dictates the values of $E \sim 10^{21} \mathrm{eV}$ and $L \sim 10^{9}$ light-years, giving $\Delta m^{2}$ reach $\sim 10^{-10}(\mathrm{eV})^{2}$ and $\Delta \xi^{(7)}$ reach $\sim 10^{-3}$. Thus, flavor oscillating neutrinos with energy in the range of $\mathrm{ZeV}$ provided they have originated at a cosmological distance of some $10^{9}$ light-years - are capable of probing the effects of quantum gravity that may be as minute as, say, septically suppressed by the Planck energy.

\section{PROSPECTS FOR OBSERVATIONS IN THE MEDIAN FUTURE}

It is clear from the above discussion that, at least in principle, ultra-high-energy neutrinos of cosmic origin can serve as a remarkably sensitive probe of the Planck regime. In fact, within the next decade, thanks to the surge of progressively larger neutrino detectors, even in practice this intriguing possibility may become reality.

To be sure, as yet no extraterrestrial neutrino of energy greater than a few tens of $\mathrm{MeV}$ has been observed by any of the existing detectors. On the other hand, as we saw above, the energy range of neutrinos necessary to probe the Planck regime may be between $10 \mathrm{PeV}$ to $\mathrm{ZeV}$, with the corresponding propagation distance ranging from $10^{5}$ light-years to $10^{10}$ light-years. This gap in energy is quite likely to be closed within a decade, however, for some of the neutrino detectors presently under construction are indeed expected to detect significant fluxes of neutrinos of energy up to and beyond $\mathrm{ZeV}$, originating from giant astrophysical sources such as active galactic nuclei (AGN) and gamma ray bursters (GRBs). Moreover, the atmospheric neutrino background of Earth happens to have such a large spectral index that, for neutrino detections in the energy region above $10 \mathrm{PeV}$, it is unlikely to sig- 
nificantly obscure the cosmogenic neutrino flux, regardless of its source [35, [36]. Furthermore, since neutrinos are neutral and stable particles, they point back to their sources, thereby providing vital information about their propagation distances (in terms of cosmological redshifts; cf., 37]). Consequently, the next generation of neutrino detectors may well be able to witness quantum gravity effects, at least of the kind discussed above.

There are a variety of different cosmogenic neutrino detectors under construction at present, or planned to be operational within the next decade (for an introductory survey, see, e.g., Ref. [38]). For instance, IceCube - a cubic kilometer size detector under construction at the South Pole [28] - is expected to be fully operational by the year 2009. It is optimized for the energy range of $10^{11}$ to $10^{18} \mathrm{eV}$, but will be sensitive to energies up to $10^{20} \mathrm{eV}$ 35. Two other projects for large neutrino detectors are under construction in the middle of the Mediterranean, namely, ANTARES [18] and NESTOR [39], not to mention the existing underwater detector located at a depth of 1100 meters in the Siberian Lake Baikal [40]. All three of these projects envisage larger cubic kilometer size extensions, with operational capacities comparable to those of IceCube, or its predecessor AMANDA [41].

The typical optimized energy range of the above underwater or under-ice detectors is from $\mathrm{TeV}$ to $\mathrm{PeV}$, with sensitivity extendible up to $\sim 10 \mathrm{EeV}$. Recently, there has been a renewed impetus for constructing detectors that can study neutrinos of super-EeV energies, with sensitivity of some of them ranging well beyond the $\mathrm{ZeV}$ scale. These include Auger [42], SAUND [3], RICE 44], ANITA [29], GLUE [45], OWL [46], and ASHRA [36, 47]. For example, ANITA experiment is planned for a 30 day balloon flight over Antarctica as early as in 2007, and expected to collect a significant flux of ultra-high-energy neutrinos. Of much wider scope is a relatively new proposal, ASHRA, which purports to conduct an "entirely all-sky survey" by a telescopic array - based near three mountain peaks in the Big Island of Hawaii-that will explore neutrinos of energy up to, and beyond, several $\mathrm{ZeV}$.

The above list of neutrino detectors is by no means exhaustive, nor does it purport to do justice to their true potential. But it does give a good indication of the intense efforts that are underway for detecting the ultra-high-energy cosmogenic neutrinos. In general, however, these efforts do have to face a major difficulty. Due to their astronomically long propagation lengths, formidable challenges lay ahead in collecting statistically significant flux of such cosmogenic neutrinos, especially of those with energies above the threshold of PeV. In spite of these potential difficulties, however, many experimental collaborations are expecting to detect significant fluxes of cosmogenic neutrinos within the next decade. For example, the ASHRA Collaboration mentioned above is expecting to detect more than 1300 events per year - at the threshold energy of $10^{19} \mathrm{eV}$ - once their telescopic array becomes fully operational. Apart from the increasingly innovative detection techniques, such optimistic expectations stem largely from the theoretical estimates obtained so far for the initial flux of neutrinos from the cosmologically distant sources [48, 49].

Ultra-high-energy neutrinos from sources such as AGN and GRBs are usually thought to be produced as secondaries of cosmic ray protons interacting with ambient matter and photon fields (for a review, see, e.g., Ref. [50]). Such proton-proton and proton-photon interactions produce neutral and charged pions, which, in turn, decay into neutrinos via the chain: $\pi^{+} \rightarrow \mu^{+} \nu_{\mu} \rightarrow e^{+} \nu_{e} \bar{\nu}_{\mu} \nu_{\mu}$. From inception, these interactions have been thought to provide a "guaranteed" source of cosmogenic neutrinos 51]. Moreover, although the absolute flux of the different flavor states of such neutrinos is presently unknown, the above decay chain strongly suggests their relative flux ratios $\phi_{\nu_{e}}^{S}: \phi_{\nu_{\mu}}^{S}: \phi_{\nu_{\tau}}^{S}$ at the source to be $\frac{1}{3}: \frac{2}{3}: \frac{0}{3}$. (Here the flux of tau neutrinos - produced in the decay chain of charmed mesons in the same reaction - is thought to be suppressed well below the order of $10^{-3}$ ). Implications of these initial flux ratios (in the literature usually referred to as "the standard ratios") have been studied extensively in recent years, especially in the context of neutrino oscillations [52, 53, 54]. However, these studies are based on a simplifying assumption that the propagation distance $L$ of such neutrinos is astronomically large compared to the oscillation length $L_{O}$; or, equivalently, that the relative quantum phase (14) acquired by the propagating masseigenstates is oscillating very rapidly. Consequently, in the scenarios considered in these studies, the neutrinos arriving at a terrestrial detector would be in an incoherent mixture of mass-eigenstates, with the above initial flux ratios reduced to $\phi_{\nu_{e}}^{D}: \phi_{\nu_{\mu}}^{D}: \phi_{\nu_{\tau}}^{D} \sim \frac{1}{3}: \frac{1}{3}: \frac{1}{3}$.

By contrast, the relation $L \sim L_{O}$ is intrinsic to both observability conditions, (28) and (34), derived above, and hence the quantum coherence will be maintained for neutrinos in these cases, throughout their long journey through the intervening vacuum. This coherence would then be reflected in the different flavor fluxes registered at the terrestrial detectors, which can be estimated as

$$
\phi_{\nu_{\beta}}^{D}=\sum_{\alpha=e, \mu, \tau} P_{\alpha \beta}(E) \phi_{\nu_{\alpha}}^{S},
$$

where

$$
\begin{aligned}
P_{\alpha \beta}(E) & :=P_{\nu_{\alpha} \rightarrow \nu_{\beta}}(E) \\
& =\delta_{\alpha \beta}-\sum_{j \neq k} U_{\alpha j}^{*} U_{\beta j} U_{\alpha k} U_{\beta k}^{*}\left(1-e^{-i \frac{\Delta m_{j k}^{2}}{2 E} L}\right)
\end{aligned}
$$

are the three-flavor transition probabilities, analogous to the two-flavor ones given by (11). In practice, however, these transition probabilities would have to be averaged over the redshift distribution of neutrino sources [37], in order to incorporate the effects of their evolution with respect to the corresponding cosmological epoch $z$ :

$$
\overline{P_{\alpha \beta}}(E)=\frac{\int_{0}^{z_{\max }} P_{\alpha \beta}(E, z) f_{\nu_{\alpha}}(z) d z}{\int_{0}^{z_{\max }} f_{\nu_{\alpha}}(z) d z},
$$


where $P_{\alpha \beta}(E, z)$-which can be obtained by replacing $E$ with $(1+z) E$ in the expression given by (36) - are the transition probabilities for neutrinos produced in the epoch $z$, and $f_{\nu_{\alpha}}(z):=\frac{d \phi_{\nu_{\alpha}}^{S}(z)}{d z}$ represents the redshift distribution of cosmogenic sources producing the neutrinos $\nu_{\alpha}$ (examples of $f_{\nu_{\alpha}}(z)$ have been studied in Ref. [37]).

It is clear from the above discussion that, given the standard flux ratios $\phi_{\nu_{e}}^{S}: \phi_{\nu_{\mu}}^{S}: \phi_{\nu_{\tau}}^{S} \sim \frac{1}{3}: \frac{2}{3}: \frac{0}{3}$ for active neutrinos being produced at a cosmogenic source, the flux ratios $\phi_{\nu_{e}}^{D}: \phi_{\nu_{\mu}}^{D}: \phi_{\nu_{\tau}}^{D}$ to be registered at a terrestrial detector can be estimated using the relation (35), which in turn depends on the oscillation length (15) via the transition probabilities (36), or (37). These flux ratios would be different, however, if, instead of (15), either the oscillation length (17), or (23), is respected by nature, provided the corresponding observability condition (34), or (28), is satisfied. In other words, the observed ratios would be different for predictions based on the dispersion relations (11), or (21), from those based on $E^{2}=p^{2}+m^{2}$.

\section{CONCLUDING REMARKS}

We have explored implications of some theories of quantum gravity for neutrino flavor oscillations, within the context of modified dispersion relations (11) and (21). In particular, we have obtained expressions for Planckscale-induced deviations from the standard oscillation length, implied by these modified dispersion relations, as functions of neutrino mass, energy, and propagation length. These expressions suggest that, in general, even those deviations that are quadratically suppressed by the Planck energy may become observable for ultra-highenergy neutrinos, originating at cosmological distances. In fact, for neutrinos in the highest energy range of $\mathrm{EeV}$ to $\mathrm{ZeV}$, deviations that are as minute as up to septically suppressed by the Planck energy may become observable. As a result - and since the next generation of neutrino detectors are poised to receive sizable flux of ultra-highenergy cosmogenic neutrinos, the chances of empirically verifying (or ruling out) such minute quantum gravity effects - within a decade - are quite promising.

\section{Acknowledgments}

I would like to thank Ted Jacobson for detecting an error in the references, and D. V. Ahluwalia-Khalilova and Subir Sarkar for their comments on the manuscript.
[1] C. Rovelli, "Notes for a brief history of quantum gravity" arXiv:gr-qc/0006061.

[2] G. Amelino-Camelia and J. Kowalski-Glikman (eds.), "Quantum Gravity Phenomenology", Lecture Notes in Physics (Springer-Verlag, Heidelberg, 2005).

[3] V.A. Kostelecký and S. Samuel, Phys. Rev. D 39, 683 (1989).

[4] J. Ellis, N.E. Mavromatos, and D.V. Nanopoulos, Phys. Rev. D 61, 027503 (2000).

[5] R. Gambini and J. Pullin, Phys. Rev. D 59, 124021 (1999).

[6] J. Alfaro, H.A. Morales-Técotl, and L.F. Urrutia, Phys. Rev. Lett. 84, 2318 (2000).

[7] J. Alfaro, H.A. Morales-Técotl, and L.F. Urrutia, Phys. Rev. D 66, 124006 (2002).

[8] R. Lehnert, Phys. Rev. D 68, 085003 (2003).

[9] O. Bertolami, R. Lehnert, R. Potting, and A. Ribeiro, Phys. Rev. D 69, 083513 (2004).

[10] L. Smolin, "How far are we from the quantum theory of gravity" arXiv:hep-th/0303185.

[11] G. Amelino-Camelia, J. Ellis, N.E. Mavromatos, D.V. Nanopoulos, and S. Sarkar, Nature 393, 763 (1998).

[12] T. Jacobson, S. Liberati, and D. Mattingly, Nature 424, 1019 (2003).

[13] T. Jacobson, S. Liberati, and D. Mattingly, Phys. Rev. D 67, 124011 (2003).

[14] T. Jacobson, S. Liberati, D. Mattingly, and F.W. Stecker, Phys. Rev. Lett. 93, 021101 (2004).

[15] S.M. Carroll, G.B. Field, and R. Jackiw, Phys. Rev. D 41, 1231 (1990).

[16] V.A. Kostelecký and M. Mewes, Phys. Rev. Lett. 87,
251304 (2001).

[17] G. Amelino-Camelia, Int.J.Mod.Phys. D 12, 1633 (2003).

[18] T. Montaruli [ANTARES Collaboration], Nucl. Phys. Proc. Suppl. 110, 513 (2002).

[19] T. Jacobson, S. Liberati, and D. Mattingly, "Astrophysical bounds on Planck suppressed Lorentz violation" arXiv:hep-ph/0407370.

[20] G. Lambiase, Mod. Phys. Lett. A 18, 23 (2003); Class. Quant. Grav. 20, 4213 (2003).

[21] R. Brustein, D. Eichler, and S. Foffa, Phys. Rev. D 65, 105006 (2002).

[22] J. Ellis, N.E. Mavromatos, D.V. Nanopoulos, and G. Volkov, Gen. Rel. Grav. 32, 1777 (2000).

[23] O. Bertolami and C.S. Carvalho, Phys. Rev. D 61, 103002 (2000).

[24] V. Barger, S. Pakvasa, T.J. Weiler, and K. Whisnant, Phys. Rev. Lett. 85, 5055 (2000).

[25] J.N. Bahcall, V. Barger, and D. Marfatia, Phys. Lett. B 534, 120 (2002).

[26] S. Choubey and S.F. King, Phys. Rev. D 67, 073005 (2003).

[27] V.A. Kostelecký and M. Mewes, Phys. Rev. D 70, 031902 (2004).

[28] J. Ahrens et al. [IceCube Collaboration], Astropart. Phys. 20, 507 (2004).

[29] ANITA, http://www.ps.uci.edu/ barwick/anitaprop.pdf

[30] J. Christian, Int. J. Mod. Phys. D 13, 1037 (2004) arXiv:gr-qc/0308028.

[31] B. Kayser, "Neutrino mass, mixing, and oscillation" arXiv:hep-ph/0104147.

[32] M. Beuthe, Physics Reports 375, 105 (2003). 
[33] L. Stodolsky, Phys. Rev. D 58, 036006 (1998).

[34] C. Giunti, Am. J. Phys. 72, 699 (2004).

[35] J. Alvarez-Muniz and F. Halzen, Phys. Rev. D 63, 037302 (2001).

[36] M. Sasaki, Y. Asaoka, and M. Jobashi, Astropart. Phys. 19, 37 (2003).

[37] C. Lunardini and A. Yu. Smirnov, Phys. Rev. D 64, 073006 (2001).

[38] C. Spiering, J. Phys. G 29, 843 (2003); Nucl. Phys. Proc. Suppl. 125, 1 (2003).

[39] P. Grieder et al. [NESTOR Collaboration], Nucl. Phys. B (Proc. Suppl.) 97, 105 (2001).

[40] D. Domogatski et al. [Baikal Collaboration], Nucl. Phys. B (Proc. Suppl.) 118, 363 (2003).

[41] J. Ahrens et al. [AMANDA Collaboration], Phys. Rev. Lett. 92, 071102 (2004).

[42] Pierre Auger Observatory, http://www.auger.org/

[43] J. Vandenbroucke et al. [SAUND Collaboration], "Experimental study of acoustic ultra-high-energy neutrino detection" arXiv:astro-ph/0406105.

[44] I. Kravchenko et al. [RICE Collaboration], Astropart.
Phys. 20, 195 (2003).

[45] P.W. Groham et al. [GLUE Collaboration], Phys. Rev. Lett. 93, 041101 (2004).

[46] F.W. Stecker et al. [OWL Collaboration], Nucl. Phys. B (Proc. Suppl.) 136, 433 (2004).

[47] ASHRA, http://www.icrr.u-tokyo.ac.jp/ ashra/

[48] R. Engel, D. Seckel, and T. Stanev, Phys. Rev. D 64, 093010 (2001).

[49] O.E. Kalashev, V.A. Kuzmin, D.V. Semikoz, and G. Sigl, Phys. Rev. D 66, 063004 (2002).

[50] H. Athar, "Some aspects of neutrino astrophysics", arXiv:hep-ph/0212387

[51] V.S. Berezinsky and G.T. Zatsepin, Phys. Lett. B 28, 423 (1969).

[52] H. Athar, M. Jeżabek, and O. Yasuda, Phys. Rev. D 62, 103007 (2000).

[53] D.V. Ahluwalia, Mod. Phys. Lett. A 16, 917 (2001).

[54] J.F. Beacom, N.F. Bell, D. Hooper, S. Pakvasa, and T.J. Weiler, Phys. Rev. D 68, 093005 (2003). 